\title{
Correspondence
}

\section{One wolf shot is too many}

Denmark's position as a haven for recolonizing top predators has been seriously undermined by the shooting in April of one of the first wolves to be born in the country in almost 200 years.

The Eurasian wolf (Canis lupus lupus) reappeared in Denmark before 2012 after at least eight individuals immigrated from Germany and Poland (see go.nature.com/2mfaxxz). The subpopulation is protected under the European Union's Habitats Directive.

The killing of the female wolf arose from controversy among hunters, farmers and politicians over the recolonization. It was widely publicized on social media. We call for greater political accountability and better management of the country's wolf population: at a minimum, there should be protected denning areas, sufficient economic compensation for sheep farmers and satellite tracking of wolves.

Without such measures, Denmark's role in setting the agenda for international agreements such as the United Nations Red List of Threatened Species could rightly be called into question.

Christian Sonne Aarhus University, Roskilde, Denmark. Aage K. O. Alstrup Aarhus University, Aarhus, Denmark. cs@bios.au.dk

\section{AI threat to drug development}

Artificial intelligence could help to identify more-effective candidate drugs (see Nature 557, S55-S57; 2018). However, this dream held by patients, clinicians, physicians and the public-health system could become a nightmare for the pharmaceutical industry.

The development of a clinically active ingredient generally costs hundreds of millions of euros, so the compound needs to be protected by a worldwide patent for the process to be economically feasible. A patent is granted only when a compound's application can be classified as both 'new' and 'invented'. A highly effective compound thrown up by an AI algorithm could indeed be new. Whether it is 'invented', however, is debatable. This is because the inventor might be considered as either the algorithm (so not a person) or its programmer.

It could be argued that if there is a connection between the program and the compound's structure, then it is predictable by experts and so no longer inventive. Or, if the programmer can't explain how the AI algorithm found the structure, then he or she didn't invent anything. Assigning and agreeing on intellectual-property rights will be even more complex when different parts of the process behind important discoveries involve multiple contributors.

Patent law would presumably have to be adapted, for example by acknowledging the development work through a temporary ban on imitation.

Lutz Heuer Dormagen,

Germany.

nievenheimer1@t-online.de

\section{Young scientists aim to prioritize patients}

Translational medicine helps to prevent scientific research from being wasted by focusing on the long-term benefits for patients. As early-career researchers, we want to accelerate that process - rather than waiting for senior researchers to spearhead the necessary changes.

Our international network of aspiring clinician-scientists, called Apollo, collaborates with senior scientists from the EUREKA Institute for Translational Medicine in Syracuse, Italy. We aim to learn about the goals, opportunities and challenges of translational medicine, including finding a more-efficient way to use research funding and balancing the competing interests of the different parties involved in research. We organize meetings, student workshops, mentorship programmes and an annual training programme.

We hope to develop the skills to navigate and improve the drugdiscovery pipeline. Speeding up research delivery from bench to bedside will also entail changing how scientists are evaluated, as well as promoting collaboration across disciplines (R. Benedictus et al. Nature 538, 453-455; 2016). Remi Stevelink ${ }^{\star}$, Gautam Kok* Apollo Society, University Medical Center Utrecht, the Netherlands. info@apollosociety.eu ${ }^{*}$ On behalf of the boards of Apollo Utrecht and Toronto; see www. apollosociety.eu for details.

\section{Cite the online date of publication}

With online delivery increasingly dominating scientific publishing, most long-established journals run papers in both print and online formats - but not necessarily simultaneously. This can affect how researchers are given scientific priority. In our view, scholars from all disciplines should use the earlier, online citation date, rather than defaulting to print as the traditional record.

In our experience, the time lag between the two can be as long as 6 months. This might be crucial for annual research evaluations, for instance, when a paper is published online at the end of one year and in print the year after (see, for example, A. L. Woerman et al. Acta Neuropathol. 135, 49-63; 2018, originally published online in August 2017). Timings are also key when two competing groups publish papers that report similar findings. Young scientists, in particular, need to have the publication date of their original work accurately recorded.

Using the online publication date as the primary citation would dispel such confusion. Reference lists that include dates for both publication formats and are picked up by public databases such as PubMed are a step in the right direction. Editors, authors and indexers need to work together to manage any effect on priority when publishing a paper in both formats.

Michael A. Keller Stanford University, California, USA. Stanley Prusiner Institute for Neurodegenerative Diseases, University of California, San Francisco, California, USA. michael.keller@stanford.edu

\section{Scavengers need help from IPBES}

Scavenger conservation is in jeopardy, which risks amplifying current threats to biodiversity and ecosystem services. In our view, ill-informed policies for managing carrion left over from farming, hunting and fisheries are largely to blame. We call for the Intergovernmental SciencePolicy Platform on Biodiversity and Ecosystem Services (IPBES) to help integrate such policies more effectively with their scientific implications as it draws up its 2020-30 programme (see Nature http://doi.org/cq8r; 2018).

Scavengers are in decline worldwide. Vulture populations in Asia crashed after consuming livestock carcasses that contained the anti-inflammatory drug diclofenac (J. L. Oaks et al. Nature 427, 630-633; 2004). Elephant and rhino carcasses poisoned by poachers are decimating African vultures and apex predators (D. Ogada et al. Conserv. Lett. 9, 89-97; 2016). And changes in how discards from European fisheries are managed could adversely affect endangered seabirds (A. W. J. Bicknell et al. J. Appl. Ecol. 50, 649-658; 2013).

The declines stand to compromise nutrient recycling, disease control and waste disposal, all of which contribute to public health and food security. Patricia Mateo-Tomás Research Unit of Biodiversity, Oviedo University, Mieres, Spain.

Pedro P. Olea Department of Ecology, Universidad Autónoma de Madrid, Spain.

rktespejos@gmail.com 